\title{
2. Untersuchungshaft: Beugung, Bestrafung, Vorbeugung?
}

\author{
nDie Persönlichkeit des Untersuchungsgefangenen \\ ist $\mathrm{zu}$ achten und sein Ehrgefüh] zu schonen. Im \\ Umgang mit ihm muß selbst der Anschein vermie- \\ den werden, als ob er zur Strafe festgehalten werde. \\ Die Untersuchungshaft ist so zu vollziehen, daß der \\ Gefangene keinen sittlichen oder körperlichen Scha- \\ den leidet.* \\ Nr. I Abs. 3 Untersuchungshaftvollzugsordnung
}

\section{Haftbedingungen von Roland Otto und Karl-Heinz Roth}

Ich will zu Beginn etwas zu den Haftbedingungen sagen, die diesem Prozeß bereits vorangegangen sind und daran anknüpfend einige Bemerkungen zur Funktion der Untersuchungshaft machen. Ich beziehe mich in meinen Ausführungen auf Unterlagen und Dokumentationen des Unterstützungsbüros für Roland Otto und KarlHeinz Roth in Köln, die ich in den letzten Tagen zur Verfügung gestellt bekommen habe. Gegendarstellungen sind mir nicht bekannt. Ich will drei Bereiche herausgreifen:

- den Bereich der Unterbringung

- den Bereich der Information

- den Bereich der Gesundheit.

Zunächst einmal ist festzuhalten, daß beide Betroffenen in strenger Einzelhaft gehalten wurden über längere Zeit. Hinter dem Begriff der strengen Einzelhaft verbirgt sich ein System besonderer Haftbedingungen, daß insgesamt darauf gerichtet ist, den Gefangenen von seiner Umwelt total zu isolieren, und jegliche Möglichkeit des menschlichen Kontakts innerhalb der Anstalt zu unterbinden. Das bedeutet im einzelnen: Einzelzelle, Ausschluß von allen Gemeinschaftsveranstaltungen, Einzelhofgang, Einzelbad etc. Unter diesen Bedingungen ist Roland Otto is Monate verwahrt worden und Karl-Heinz Roth ist in dieser Weise bis Ende Dezember 1976, also 20 Monate, verwahrt worden. Dann ist die Isolierung bei Karl-Heinz Roth gelockert worden und im Februar 1977 ist sie dann während des Prozesses wieder verschärft worden. Zusätzlich war die Zelle von Karl-Heinz Roth zeitweise auch nachts stark beleuchtet durch Außenscheinwerfer, die vor seiner Zelle angebracht waren. Der Isolation im Inneren der Anstalt entspricht die Isolation von der Außenwelt. Karl-Heinz Roth unterlag während der ersten Zeit seiner Haft einer besonders engherzig gehandhabten Briefkontrolle. Der Haftrichter hatte festgelegt, daß er nur 10 Seiten pro Woche ausgehende Post und 20 eingehende Post erhalten bzw. verschicken durfte, wobei eine bestimmte Schriftgröße und ein fester Zeilenabstand vorgeschrieben waren. Eine Schreibmaschine wurde Karl-Heinz Roth über ein Jahr lang vorenthalten; im August 1976 hat er dann eine solche Schreibmaschine erhalten, die aber völlig demontiert und zunächst funktionsuntüchtig war. Soviel zunächst zur Unterbringung und zur Isolationshaft.

Ein weiterer Bereich der Haftbedingungen ist der der Information. Karl-Heinz Roth ist von Mai 1975 bis Oktober 1975 ohne Zeitungen, ohne Radio, ohne Bücher außerhalb der Anstaltsbücher gehalten worden. Im Oktober 1975 hat er dann die ersten vier selbstgewählten Bücher zur Verfügung bekommen, die dann bis Dezember reichen mußten. Im Dezember wurden dann Zeitungen zugelassen, allerdings unter allerlei Hindernissen, erstens kamen sie nur sporadisch, wurden bei den 
zahlreichen Verlegungen nicht nachgeschickt und mit "Empfänger unbekannt" zurückgeschickt und zweitens die, die ankamen, waren - wie er sich ausdrückt - mehr »Fenster «, weil alle Anzeigen herausgeschnitten waren, eine Reihe Artikel herausgeschnitten waren, so daß im wesentlichen der Sportteil vollständig war. Im Dezember 1975 ist dann eine Radioerlaubnis erteilt worden, die sich aber zunächst darauf beschränkte, den Anstaltsfunk zu hören. Ein weiteres Jahr später ist ihm dann ein eigenes Radio bewilligt worden.

Uber die Krankengeschichte von Karl-Heinz Roth wäre vieles zu sagen, ich will mich hier auf einige wenige Angaben beschränken, die für mein Thema zentral sind. Karl-Heinz Roth ist mit einem Bauchschuß und mit einem Lungenschuß verhaftet worden. Das waren nur die schwersten Verletzungen. Er ist in der Haft zweimal operiert worden, es sind diverse Komplikationen aufgetreten, offenbar auch Fehldiagnosen und Fehlbehandlungen. Sogenannte Sicherheitsaspekte haben stets im Vordergrund gestanden, selbst auf den Intensivstationen war er stets von schwerbewaffneten Polizisten bewacht. Bei seinen insgesamt 16 Verlegungen von einem Anstaltskrankenhaus ins andere und auch zum Teil in die ganz normale U-Haftzelle war er meist gefesselt, manchmal sogar an Händen und Füßen gefesselt, was bei seinem Krankheitszustand ganz besonders schmerzhaft ist. Resultat: Sein gegenwärtiger Gesundheitszustand ist äußerst schlecht, weshalb auch die Anstaltsärzte ihn nur für beschränkt verhandlungsfähig erklärt haben. Die gegenwärtige Situation wird so umschrieben, daß durch diese Versorgung die untere Dickdarmschlinge soweit beschädigt ist, daß dieser Darmabschnitt keinen Darminhalt mehr transportiert. Es kommt oberhalb dieses Abschnittes zu einem Stau des Darminhalts mit spastischer Verkrampfung des Darms und Koliken, die auch in der Verhandlung inzwischen mehrfach aufgetreten sind. Karl-Heinz Roth leidet zusätzlich unter starken Kreislaufstörungen, erheblichem Untergewicht, er hat zeitweise über $30 \mathrm{~kg}$ verloren gehabt, und psychischen Deprivationen. Dennoch ist ihm über 23 Monate - und das halte ich jetzt für einen gewissen Höhepunkt der ganzen Geschichte - die Behandlung durch einen Arzt seines Vertrauens verwehrt worden. Haftverschonung ist bereits vor über einem Jahr abgelehnt worden aufgrund einer Stellungnahme des Gefängnisarztes von Köln, des gleichen Gefängnisarztes, der Herrn Herstatt schon nach 6 Wochen für haftunfähig erklärt hat. Dies war nur eine Auswahl von Fakten, die mir in den letzten Tagen erst in dieser Präzision bekannt geworden sind.

\section{Haftbedingungen und Strafprozeßrecht}

Ich will das jetzt versuchen zu kontrastieren mit dem was hier eigentlich Untersuchungshaft heißt. Wohl gemerkt, die beiden Angeklagten Karl-Heinz Roth und Roland Otto befinden sich eben nicht in Strafhaft, sondern sie befinden sich in Untersuchungshaft. Für die Untersuchungshaft gelten Bestimmungen, die von altersher sichern sollen, daß eine ordnungsgemäße Ermittlungsverfahrensabwicklung sichergestellt wird. Die Untersuchungshaft darf selbst noch keinerlei Strafe darstellen. Dieser Zweck der Untersuchungshaft ist zusammengefaßt im $\$ I 99 der Strafprozeßordnung und darin heißt es, daß nur solche Beschränkungen auferlegt werden dürften, die der Zweck der Untersuchungshaft und die Ordnung in der Vollzugsanstalt fordern. Der Zweck ist ausschließlich die Sicherung des Strafverfahrens gegen Flucht und gegen Verdunklung. Ausnahmen gelten nur für gewisse Sittlichkeitsdelikte und andere Rückfalltaten, wie sie in den letzten is Jahren Eingang gefunden haben in die Strafprozeßordnung, die hier aber nicht einschlägig 
sind. Zweck der Untersuchungshaft ist also nur gegen Flucht und gegen Verdunklung zu sichern. Jetzt ist also zu prüfen, was die aufgezählten Bedingungen der Untersuchungshaft mit Fluchtgefahr und Verdunkelung zu tun haben. Zumindest bei Karl-Heinz Roth scheidet Fluchtgefahr von vorneherein aus. Er wurde als schwerverletzt festgenommen und hat sich bis heute von seinen Verletzungen nicht erholt. Es war deshalb zu jedem Zeitpunkt absurd, sich diesen Menschen, der einer intensiven Behandlung und vermutlich weiterer Operationen bedarf, als Flüchtling vorzustellen. Hinzu kommt, daß Karl-Heinz Roth als auch Roland Otto sehr deutlich und sehr glaubhaft versichert haben, daß sie an der Durchführung dieses Prozesses selbst intensiv interessiert sind. Die Informationssperre, der Entzug von Radios, Zeitschriften und Zeitungen, Büchern hat sicherlich nichts mit der Verhinderung einer Flucht zu tun, ebenso wenig wie die totale Isolation. Das wird auch ganz deutlich durch die Tatsache, daß diese Maßnahmen nach vielen Monaten graduell zum Teil gelockert, zum Teil aufgehoben wurden. Dies also zur Fluchtgefahr. Die Verdunklungsgefahr tritt argumentativ immer dann ein, wenn Fluchtgefahr nicht so eindeutig bewiesen werden kann. Das Kriterium Verdunkelungsgefahr ist ein nahezu alles abdeckender Zweck der U-Haft. Dazu ist zu sagen, daß dieser Gesichtspunkt doch immerhin ernst zu nehmen ist, da bei dem Ereignis in Köln am 9. Mai 1975 zwei Menschen ums Leben gekommen sind, und es mag durchaus ein Interesse bestehen, etwa Zeugenaussagen abzusprechen und Zusammenhänge zu verdunkeln. Dieses Interesse hingegen mag genauso bei den beteiligten Polizeibeamten vorliegen, die dazu seit 23 Monaten jede Gelegenheit hatten. Hinzu kommt, daß man Freiheitsbeschränkungen nicht unbegrenzt auf Verdunklungsgefahr stützen kann, es muß auch noch etwas zum Verdunkeln da sein und dazu hat der ehemalige Bremer Generalstaatsanwalt Dünnebier geschrieben*", daß die Verdunklungsgefahr endet, wenn die Ermittlungen beendet sind, sie besteht nur so lange, wie es noch etwas zu verdunkeln gibt. Er sagt: alle Beweise in Haftsachen können in der Regel in 3 Monaten gesichert werden mit Ausnahme von Sachverständigenbeweisen und diese wiederum sind gegen die Einwirkungen des Beschuldigten immun. Verdunklungsgefahr ist also ein außerordentlich schwammiger Begriff, von dem Praktiker wie Dünnebier sagen, nach 3 Monaten gibt es in der Regel nicht mehr viel zu verdunkeln. Roth ist dagegen seit 23 Monaten in Untersuchungshaft. Es handelt sich darüber hinaus in diesem Fall auch nicht um besonders komplizierte Ermittlungen, wie sie etwa in Wirtschaftsstrafsachen durchaus vorkommen mögen, sondern es geht um einen einzigen Vorfall, der sich am 9. Mai 1975 innerhalb weniger Minuten ereignet hat, dazu sind die Spuren zu sichern, dazu sind die Beteiligten zu hören, das sind die wesentlichen Ermittlungen, die hier zu führen sind. Eine Verlängerung der Untersuchungshaft über diesen Zeitraum hinaus ist lediglich dazu angetan, das Erinnerungsproblem zu vergrößern.

\section{Funktion der Haftbedingungen}

Wenn demnach weder Flucht- noch Verdunklungsgefahr die Länge der Untersuchungshaft und die Haftbedingungen rechtfertigen können, wodurch sind diese dann zu erklären? Ich meine, daß die U-Haft hier in diesem wie auch in anderen und nicht nur politischen Fällen illegal mißbraucht wird zur Beugung, zur Bestrafung und zur Vorbeugung. Dies sei noch kurz erläutert.

" Hanns Dünnebier, Reform der Untersuchungshaft, in: Hans Lüttger (Hrsg.), Probleme der Strafprozeßreform, Berlin 1975, S. 32. 
Der Beschuldigte hat nach deutschem Strafprozeßrecht das Recht, die Aussage zu verweigern, insbesondere die Aussage zur Sache erst in der Hauptverhandlung zu machen, wie es in diesem Fall auch geschehen ist. Es ist durchaus illegal, zu versuchen, durch Isolation, Informationssperre und durch immer weitere Ausdehnung der U-Haft eine Aussage oder ein Geständnis des Inhaftierten erreichen zu wollen. Anhand von Materialien aus politischen Prozessen ist dieser Beugecharakter der U-Haft schon vor einigen Jahren demonstriert und analysiert worden*. Ubrigens bezieht sich der Beugecharakter der U-Haft meist nicht nur auf die Aufklärung der Ausgangstat, sondern der Beugecharakter bezieht sich meist noch auf ganz andere Taten, und dies nicht nur bei politischen Tätern: es ist gängige Polizeipraxis, $\mathrm{daß}$ man versucht, einen Menschen, den man einmal in Haft hat, in Situationen zu halten, von denen die Verfolgunsbehörden sich Aufklärung auch in anderen Zusammenhängen versprechen. Dies ist erst recht nicht vom Zweck der Untersuchungshaft gedeckt.

Über den Beugecharakter der U-Haft hinaus kommt dieser auch eine Bestrafungsfunktion zu, das ist auch im Fall Roth und Otto deutlich. Dort, wo die Unbeugsamkeit eines Häftlings sich erwiesen hat, geraten alle Zwangsmaßnahmen zur Strafe für seine Tat. Denn an die Stelle der Unschuldsvermutung ist hier die Schuldvermutung getreten. Auch dies ist ein Phänomen, das sich in ganz unpolitischen Verfahren zeigt. Wer erst einmal in U-Haft sitzt, wird als Schuldiger vermutet, was lediglich im einzelnen noch zu beweisen ist. Daß dieser Bestrafungscharakter der U-Haft vielfach härter und grausamer als der normale Strafvollzug ist, zeigt sich ganz besonders deutlich am Fall Roland Otto, weil Roland Otto nach dem er ein Jahr lang in Untersuchungshaft war eine Restfreiheitsstrafe aus einem vorherigen Verfahren angetreten hat, er damit aus der Untersuchungshaft in der Strafvollzug verlegt wurde und damit eine Reihe von Beschränkungen der Untersuchungshaft aufgehoben worden sind.

Schließlich soll dem als schuldig vermuteten Verdächtigen auch noch "das Handwerk gelegt " werden. Ein Teil der Haftbedingungen ist nur erklärbar aus diesem Bestreben der Verfolgungsorgane, denkbare "weitere" Straftaten zu verhindern. Hierher gehört etwa die Unterbindung von Kontakten zu "Gesinnungsgenossen " weit über das zur Verhinderung von Verdunklung erforderliche Maß hinaus. Auf diese Weise feiert die im Gesetzgebungsverfahren gescheiterte Vorbeugehaft in der Praxis der Untersuchungshaft fröhliche Urstände.

Mir kam es darauf an, bei aller Unterschiedlichkeit auf Gemeinsamkeiten zwischen "politischen « und »unpolitischen " Fällen hinzuweisen. In der Untersuchungshaft scheint es mir heute in der Bundesrepublik durchaus die Regel zu sein, daß ein Rechtsbruch stattfindet, daß die Bestimmungen des $\$$ i I 9 der Strafprozeßordnung weit überschritten werden. Ich meine, daß dem Einhalt geboten werden muß. Im Falle Karl-Heinz Roth und Roland Otto kann die Forderung von Anfang an nur gewesen sein, die Angeklagten aus der Haft zu entlassen. Darüber hinaus müssen wir nach Wegen und Formen suchen, wie auch dem unbekannten Untersuchungsgefangenen zu seinen verbrieften Grundrechten zu verhelfen ist.

Johannes Feest

*. Klaus Eschen u. a., Folter in der BRD, Dokumentation zur Lage der politischen Gefangenen, zusammengestellt von Verteidigern in politischen Strafsachen, in: Kursbuch 32, 1973 , S. $66 \mathrm{f}$. 
Liebe Kommilitoninnen und Kommilitonen, liebe Kollegen. Ich bin von den Veranstaltern gleichsam als Spezialist für das Allgemeine engagiert worden, ich soll einige tiefschürfende Aussagen über politische Gefangene im politischen Prozeß und in der politischen Justi : machen. Gleichzeitig habe ich konzediert, daß ich nur 20 Minuten sprechen darf. Damit habe ich mir die Quadratur des Kreises auferlegt, der ich mich aber nicht unterziehen will, ich werde mich nur auf einen Aspekt beschränken. Anknüpfend an Johannes Feest, der da sagte, daß die U-Haft eine vorweggenommene Strafe sei für die Tat, möchte ich sagen, daß ich das etwas anders sehe. Es ist dies ja nicht die Strafe für die Tat, sondern die Strafe für den Täter oder für die Gesinnung des Täters. Zwei Dinge sind bei der U-Haft zu berücksichtigen: 1. Es ist eine vorläufige Maßnahme, die nicht bereits Vollstreckungscharakter haben darf. Die höchste Entfaltung dieser Paradoxie, daß U-Haft eine Strafe ist, daß eine vorläufige Maßnahme zu einer endgültigen Maßnahme wird, liegt dann vor, wenn U-Haft dazu führt, daß jemand den Tod findet.

2. Der andere Gesichtspunkt ist der, ob es sich tatsächlich um eine Strafe für eine Tat handelt, d. h. also ein historisches Ereignis, das durch Zeugen und sonstige Sachmittelbeweise umgrenzt und umschrieben wird, oder ob es nicht doch mehr ist, nämlich die Bestrafung für eine bestimmte Gesinnung. Dies ist der Gesichtspunkt, den ich kurz entwickeln will, weil er nicht nur für diesen Prozeß gilt, sondern weil er doch paradigmatisch ist für eine ganze Reihe von Prozessen, und der schon bestimmte institutionelle Veränderungen, auch im Bundeskriminalamt, auf die ich noch zu sprechen komme, zur Folge hatte. Zunächst einmal ist auffällig, daß noch bis vor kurzem der Begriff des politischen Gefangenen, des politischen Täters, des politischen Straftäters von den herrschenden Repräsentanten dieses Landes nicht gerne gehört wurde. Es ist immer wieder betont worden, es handele sich um ganz gemeine Kriminelle: Räuber, Mörder, Geiselnehmer oder was auch immer. Während umgekehrt die Betroffenen, das heißt die in diesen Angelegenheiten Inhaftierten, auf dem politischen Charakter ihrer Taten bestanden.

So wird es auch heute noch zum Teil vertreten: ich habe gerade gestern in der Frankfurter Allgemeinen Zeitung gelesen, der Bundesjustizminister Vogel habe betont, es gäbe gar keine politischen Täter, sondern das seien alles ganz normale Kriminelle. Gleichzeitig wurde aber anläßlich des Attentats auf Buback immer wieder deutlich erklärt, es handele sich um ein Attentat auf den Rechtsstaat, was ja eine ungemein politische Erklärung ist, denn das bedeutet, ein Attentat nicht auf eine physische Person, ein lebendiges Wesen, sondern auf ein Prinzip, auf ein politisches Prinzip, das Prinzip Rechtsstaat. Das ist dann im Bewußtsein derer, die dieses aussprechen, ein politisches Delikt. Diese Widersprüchlichkeit zu untersuchen, wäre sicherlich sehr reizvoll, ich hatte auch erst die Absicht, dies hier zu tun, aber das wäre hier etwas zu kompliziert geworden. Aber, es hat mir doch Anlaß gegeben zu fragen, wie es eigentlich dazu kommt, daß jetzt der politische Charakter von Taten und die politische Motivation von Tätern im Verhältnis zu der Zeit vor etwa zwei, drei Jahren, jetzt auch von Repräsentanten des Staates, wenn auch in widersprüchlicher Weise akzeptiert wird.

Hier finden wir nun eine fatale Wendung der Funktionalisierung des Begriffs des politischen Straftäters, der einmal nur besagen sollte, daß der Einzelne nicht etwa privilegiert werden sollte durch das herrschende Strafrecht, sondern daß er lediglich in den ihm gemachten politischen Prozessen ganz bestimmte Rechte haben müsse, um sich angemessen verteidigen zu können. $\mathrm{Daß}$ man ihm den politischen Straftäter 